\title{
GESTÃO EM SAÚDE E O USUÁRIO DO PROGRAMA FARMÁCIA POPULAR DO BRASIL COM DIABETES DIAGNÓSTICA
}

\author{
A.S.D.G. da Silva*, D.F. Lima* e S.B.Franco*, M.M.F.Gomes*, R.G.G.Amorim*
*Universidade de Brasília, Faculdade Gama (UNB/FGA), Programa de Pós-Graduação em Engenharia Biomédica, Brasília, Brasil
e-mail: alberluciadamaso@gmail.com

\begin{abstract}
Resumo: Apresentamos neste trabalho os resultados de um estudo descritivo sobre o perfil dos usuários do Programa Farmácia Popular do Brasil (PFPB) com diabetes Os dados utilizados são oriundos da Pesquisa Nacional de Saúde (PNS) realizada em 2013. O principal resultado deste trabalho evidencia que $57,19 \%$ dos indivíduos diagnosticados com diabetes não obtém o medicamento via o Programa Farmácia Popular do Brasil.
\end{abstract}

Palavras-chave: Pesquisa Nacional de Saúde, Diabetes, Programa Farmácia Popular do Brasil.

Abstract: In this work, we show the results of a descriptive study about characteristics of users of Popular Pharmacy of Brazil Program (PPBP) who have diabetes. The data used are from National Health Research (NHR) held in 2013. The main result shows that $57,19 \%$ of individuals diagnosed with diabetes not get the medicament by popular pharmacy of Brazil program.

Keywords: National Health Research, Diabetes, Popular Pharmacy of Brazil Program.

\section{Introdução}

No Brasil, a saúde é um direito estabelecido na Constituição Federal de 1988 que, no âmbito público, é garantida por meio de políticas sociais consolidadas por meio do Sistema Único de Saúde (SUS), o qual foi institucionalizado pela Lei 8.080 , de 19 de setembro de 1990, sendo regida pelos princípios da universalidade, igualdade no atendimento, integralidade das ações em saúde, dentre outros [1]. As ações do SUS estão inseridas no contexto atual da gestão de tecnologias em saúde como o conjunto de atividades gestoras relacionado com os processos de avaliação, incorporação, difusão, gerenciamento da utilização e retirada de tecnologias do sistema de saúde.

A Engenharia Biomédica é uma grande aliada das chamadas Avaliações de Tecnologias em Saúde (ATS), pois ela promove o gerenciamento dos serviços em Saúde que vão desde os medicamentos, equipamentos, procedimentos e os sistemas organizacionais até o suporte dos cuidados com a saúde. O conceito de tecnologia em saúde abrange qualquer intervenção que pode ser utilizada para promover a saúde e bem estar das pessoas, e para desenvolver esse trabalho faz-se fundamental a participação do engenheiro biomédico. [2].
Acreditando em uma gestão eficaz com o uso das ATS, o Governo Federal desenvolve através do Ministério da Saúde (MS) em todo Brasil, a assistência farmacêutica, por meio da atenção básica à saúde com distribuição de alguns medicamentos de uso contínuo nos Postos de Saúde. Os medicamentos para diabetes e hipertensão, por exemplo, fazem parte desse contexto. E com o aumento dos casos de diabetes, o governo decidiu implantar o Programa Farmácia Popular do Brasil (PFPB) [3] [4]. Os indivíduos portadores de hipertensão e diabetes por sua vez são cadastrados pelas Secretarias de Saúde através do Sistema de Cadastramento e Acompanhamento de Hipertensos e Diabéticos (HIPERDIA) [5].

Nesse arcabouço, um problema de saúde que afeta um grande quantitativo de brasileiros e cuja prevenção, diagnóstico e tratamento encontra obstáculos. A diabetes é classificada como uma doença crônica não transmissível. Quase um quarto dos brasileiros adultos tem que enfrentar a diabetes, mas com um maior controle da doença tem diminuído fortemente o número de complicações ligadas à doença [1] [5].

Com o propósito de conhecer a ação da gestão em tecnologias da saúde e o usuário do Programa Farmácia Popular do Brasil com diabetes diagnosticada, desenvolvemos esse trabalho com base nos dados da PNS 2013.

\section{A gestão em Saúde e papel do engenheiro biomédico}

Com o objetivo de ampliar o acesso da população às tecnologias, a Agência Nacional de Vigilância Sanitária (ANVISA) tem atuado desde 2000 no campo da regulação econômica de medicamentos. Porém, foi a partir de 2003, com a criação de uma unidade organizacional dedicada à área de avaliação econômica de tecnologias em saúde, que a Agência passa a ter uma atuação mais forte na área de Avaliação de Tecnologias em Saúde (ATS) [2]. Em 2003 foi instituído pela Portaria $\mathrm{n}^{\mathrm{o}} 1.418 / \mathrm{GM}$ de 24 de julho de 2003 o Conselho de Ciência, Tecnologia e Inovação do Ministério da Saúde (CCTI/MS). Este Conselho, coordenado pela SCTIE, possui entre as suas atribuições: implantar a Política de Ciência, Tecnologia e Inovação em Saúde; definir diretrizes e promover a avaliação de tecnologias visando à incorporação de novos produtos e processos pelos gestores, prestadores e profissionais dos serviços de saúde. Assim, por meio da 
Lei 12.401, de 28 de abril de 2011, o SUS implementou a Comissão Nacional de Incorporação de Tecnologias (CONITEC) no sentido de qualificar o processo de incorporação, exclusão ou alteração dos medicamentos e outras tecnologias no âmbito do sistema de saúde no Brasil, a partir das análises críticas da literatura disponível sobre a eficácia, efetividade, segurança e custos dessas tecnologias [2][6].

Neste cenário o engenheiro biomédico é fundamental na gestão dos insumos em Saúde, pois a Engenharia Biomédica aplica princípios da engenharia elétrica, mecânica, química, óptica e outros, para entender, modificar, ou controlar sistemas biológicos. Quando um engenheiro biomédico trabalha dentro de um hospital ou clínica, ele é mais propriamente chamado de engenheiro clínico. $\mathrm{O}$ engenheiro clínico é apresentado como o responsável pela interface entre o corpo clínico, os administradores hospitalares, os fornecedores e as agências reguladoras, sempre com o objetivo de garantir que a tecnologia médico-hospitalar seja utilizada de forma efetiva e segura [7].

\section{Programa Farmácia Popular do Brasil (PFPB) e uso de medicamentos para diabetes}

O Programa Farmácia Popular do Brasil, foi criado em 2004 pelo Governo Federal, em associação com a Fundação Osvaldo Cruz (Fiocruz), uma instituição que tem a responsabilidade de disponibilizar os medicamentos mediante ressarcimento, assegurando à população o acesso a produtos básicos e essenciais à saúde a um preço reduzido, pela Lei no 10.858 , de 13 de abril de 2004, regulamentada pelo Decreto no 5.090, de 20 de maio de 2004 [3] [8]. O PFPB tem como o objetivo principal garantir o acesso a medicamentos para todos os usuários, mediante ressarcimento dos custos; tanto para os que possuem plano de saúde privado, como também aos usuários do Sistema Único de Saúde (SUS) que não encontrarem medicamentos disponíveis na Farmácia Básica [4].

Em 2006 o programa foi expandido, também mediante parcerias, desta vez com farmácias e drogarias privadas e facilitou o acesso a medicamentos essenciais. Para adquirir, basta que o interessado visite uma unidade própria do programa ou uma drogaria com a marca "Aqui tem Farmácia Popular" [8]. Nas unidades próprias, é necessária a apresentação da receita médica ou odontológica. Nas drogarias, além da receita é necessária a apresentação do comprovante de endereço, documento com foto e o CPF. Para cada pessoa, será entregue medicamento suficientes para um mês [3].

Os medicamentos do PFPB devem estar disponíveis em todos os momentos em quantidades e dosagens adequadas e a um preço que os indivíduos e a comunidade possam pagar. A ideia de medicamentos essenciais existe em todo o mundo e compreende uma lista de medicamentos que satisfazem às principais.

Necessidades do cuidado de saúde da população. Esses medicamentos são selecionados por critérios de eficácia, isto é, se funcionam corretamente, de segurança, de conveniência, de qualidade e comparação de custo mais favorável [4].

É importante ressaltar que o PFPB tem contribuído para o controle e diminuição das internações dos pacientes diagnosticados com diabetes no Brasil [10].

O diabetes mellitus é uma síndrome caracterizada pelo excesso de açúcar (glicose) no sangue. Esta síndrome é decorrente da deficiência total ou parcial da produção de insulina (hormônio produzido pelo pâncreas) ou ainda, quando a insulina produzida não consegue agir. O diabetes quando não diagnosticado ou se não tratado adequadamente, pode levar a complicações, o que torna esta doença um grave problema de saúde pública [11].

Uma epidemia de diabetes mellitus (DM) está em curso. Atualmente, estima-se que a população mundial com diabetes seja da ordem de 387 milhões e que alcance 471 milhões em 2035[11] [12].

O número de diabéticos está aumentando em virtude do crescimento e do envelhecimento populacional, da maior urbanização, da progressiva prevalência de obesidade e sedentarismo, bem como da maior sobrevida de pacientes com DM quantificar o predomínio atual de DM e estimar o número de pessoas com diabetes no futuro é importante, pois possibilita planejar e alocar recursos de maneira racional [11] [13].

Dados brasileiros de 2011 mostram que as taxas de mortalidade por DM (por 100 mil habitantes) são de 33,7 para a população geral, 27,2 nos homens e 32,9 nas mulheres, com acentuado aumento com o progredir da idade, que varia de 0,50 para a faixa etária de 0 a 29 anos a 223,8 para a de 60 anos ou mais, ou seja, um gradiente de 448 vezes [11] [14].

\section{Materiais e métodos}

A pesquisa oriunda deste artigo é classificada como um estudo descritivo e exploratório, utilizando dados da PNS de 2013.

A Pesquisa Nacional de Saúde (PNS) é uma pesquisa de base domiciliar, de âmbito nacional, com amostra de 80.000 domicílios em 1.600 municípios, realizada em parceria com o Instituto Brasileiro de Geografia e Estatística (IBGE). A pesquisa foi planejada para ser representativa para Brasil, Grandes Regiões, Unidades Federativas, capitais, áreas urbanas e rurais [4]. Porém efetivamente foram entrevistados 64.348 domicílios, sendo que 60.202 moradores responderam as questões relativas ao seu estilo de vida, estado de saúde e doenças crônicas. Os detalhes acerca da técnica de amostragem utilizada estão disponíveis no relatório sobre os resultados da PNS [15].

Os inquéritos populacionais de saúde, além de levantarem informações importantes para avaliação de saúde da população e seus estilos de vida, permitem a verificação de indicadores de desempenho do sistema de saúde do ponto de vista do usuário, de modo a subsidiar a formulação, o monitoramento e a avaliação das políticas públicas de saúde [4].

Nesse sentido, abordamos duas questões 
pertencentes ao módulo Q do questionário da PNS questões relativas às doenças crônicas, quais sejam: Q030 e a Q036. Essas perguntas indagavam ao entrevistado o seguinte:

Q030: Algum médico já lhe deu o diagnóstico de diabetes? 1.Sim; 2.Apenas durante a gravidez; 3.Não

Q036: Algum dos medicamentos para diabetes ou insulina foi obtido no Programa Farmácia Popular do Brasil (PFPB)? 1.Sim, todos; 2.Sim, alguns; 3. Não, nenhum

As variáveis explicativas que foram levadas em consideração no estudo estão relacionadas, à idade (variável quantitativa), sexo, cor ou raça, escolaridade, estado civil e região (variáveis qualitativas).

Dentre todas as questões elencadas na PNS, essas foram escolhidas como objeto do nosso trabalho, com a pretensão de elaborar um mapeamento descritivo do perfil do usuário da Farmácia Popular com diabetes, e em contrapartida estabelecer uma correlação entre os indivíduos com tal doença crônica diagnosticada e a utilização dos medicamentos para pressão alta oriundos do PFPB. Com isso, os dados analisados foram obtidos no banco de dados da PNS realizada em 2013, cujas informações e dados estão disponíveis em http://www.pns.icict.fiocruz.br/ e também na referência [15].

As análises dos dados foram realizadas pelo software Statistical Package for the Social Sciences (SPSS) versão IBM 19 [11] [12].

\section{Resultados}

Na Pesquisa Nacional de Saúde realizada em 2013 foram entrevistados 205.546 indivíduos, dos quais 3.838 são diabéticos e apenas 1.643 fazem uso de medicamentos ou insulina do PFPB. A Tabela 1 mostra que esse subgrupo populacional é caracterizado principalmente por indivíduos do sexo feminino (68\%), com 60 anos ou mais $(53,50 \%)$, que declararam cor/raça como não branca $(60,30 \%)$, de baixa escolaridade e casados $(44,20 \%)$. As regiões Sudeste $(30,50 \%)$ e Nordeste $(28,70 \%)$ detém a maioria desses usuários.

Observando os dados contidos na Tabela 1, pode-se tecer algumas observações relevantes. A primeira delas é em relação ao sexo dos entrevistados pela PNS, onde há uma predominância de mulheres. Em relação à cor da pele, o número de indivíduos que se declararam nãobrancos é maior que daqueles que se declararam brancos. $\mathrm{Na}$ distribuição por região geográfica, tem-se que a maior parte dos indivíduos entrevistados concentra-se na região Sudeste, seguida pela região Nordeste e Centro-Oeste.

O principal resultado deste trabalho, merecedor de destaque, diz respeito ao percentual, dentre aqueles com diabetes diagnosticada, de indivíduos que obtiveram algum medicamento utilizando o PFPB. Neste escopo, 42,81\% (1643) dos entrevistados afirma ter obtido pelo menos um dos medicamentos ou insulina para diabetes a partir do PFPB. A maior parte deles, 57,19\%, nunca obteve nenhum dos medicamentos ou insulina a partir do programa do governo federal.

\begin{tabular}{|c|c|c|}
\hline \multicolumn{2}{|c|}{ Características } & $\%$ \\
\hline \multirow[t]{2}{*}{ Sexo } & Homem & 32,00 \\
\hline & Mulher & 68,00 \\
\hline \multirow{3}{*}{ Idade } & 18 a 24 anos & 0,60 \\
\hline & 25 a 59 anos & 45,90 \\
\hline & 60 a 95 anos & 53,50 \\
\hline \multirow{2}{*}{$\begin{array}{c}\text { Cor ou } \\
\text { Raça }\end{array}$} & Branca & 39,70 \\
\hline & Não Branca & 60,30 \\
\hline \multirow{6}{*}{ Escolaridade } & Não-Alfabetizados & 20,00 \\
\hline & Alfabetização & 34,40 \\
\hline & Ensino Fund. & 17,50 \\
\hline & Ensino Médio & 21,80 \\
\hline & Graduação & 6,10 \\
\hline & Pós-Graduação & 0,20 \\
\hline \multirow{4}{*}{ Estado Civil } & Casado & 44,20 \\
\hline & Separado/Divorciado & 10,90 \\
\hline & Viúvo & 23,30 \\
\hline & Solteiro & 21,60 \\
\hline \multirow{5}{*}{ Região } & Norte & 13,50 \\
\hline & Nordeste & 28,70 \\
\hline & Sudeste & 30,50 \\
\hline & Sul & 13,50 \\
\hline & Centro-Oeste & 13,80 \\
\hline \multicolumn{2}{|c|}{ Tamanho da Amostra } & 1643 \\
\hline \multicolumn{3}{|c|}{$\begin{array}{l}\text { Tabela 1- Perfil dos usuários do Programa Farmácia Popula } \\
\text { do Brasil (PFPB) diagnosticados com diabetes, Brasil, } 2013 \\
\text { (Elaborado pelos autores com base nos dados da PNS, acesso } \\
\text { em junho, 2016) }\end{array}$} \\
\hline
\end{tabular}

\section{Discussão}

Ao analisarmos os dados adquiridos para esse trabalho percebe-se que a abrangência da PFPB não se apresenta de modo satisfatório, de maneira que algumas hipóteses podem ser levantadas, dentre as quais, destacam-se como principais: o fato de o diagnosticado com diabetes nunca ter obtido o medicamento via o PFPB pode ter relação com algum problema de divulgação ou publicidade do programa; os medicamentos podem estar mal distribuídos por regiões.

Sendo assim, na tentativa responder a tais hipóteses, um estudo com mais detalhes deve ser realizado, destacando as minucias regionais dos individuos portadores de necessidade por medicamentos. Com essas pesquisas, obtém-se não apenas o melhoramento do PFPB, como também um tratamento mais adequado para o portador de diabetes.

\section{Conclusão}

Os resultados da PNS sugerem que aproximadamente $6,2 \%$ da população brasileira com 18 anos ou mais de idade referiram diagnóstico médico de diabetes, sendo de 7,0\% nas mulheres e de 5,4\% nos homens. Porém, destes indivíduos diagnosticados, apenas $42,81 \%$ faz uso do PFPB para obter algum dos medicamentos ou insulina. Esse percentual é considerado pequeno, haja vista que esse programa 
proposto pelo governo federal foi idealizado para ser abrangente. Nota-se então a necessidade de se organizar estudos mais detalhados, cujo objetivo seja verificar se dentro do programa há falhas de alocação e distribuição de tais medicamentos.

Com as informações contidas neste trabalho espera-se que, os 3.838 indivíduos diagnosticados com diabetes consiga ter acesso aos medicamentos oferecidos pelo PFPB, uma vez que apenas 1.643 fazem uso do mesmo.

Os trabalhos já publicados a respeito do PFPB com base na PNS relacionados a diabetes, não fazem menção a ATS, nem correspondência ao Engenheiro Biomédico nem tão pouco trata do perfil do usuário.

O trabalho mais recente publicado pela revista Epidemiol. Serv. de Saúde em jan-março de 2016 trata apenas da obtenção de medicamentos através do PFPB, e abrange tanto hipertensos como diabéticos.

Enfim, conhecer esse perfil do usuário do PFPB é importante para a gestão do programa, tanto em relação ao indivíduo, quanto do território. E essa gestão encontra-se também como incumbência do Engenheiro Biomédico que trabalha na melhoria da Avaliação de Tecnologia em Saúde (ATS).

\section{Referências}

[1] Brasil. Lei n ${ }^{\circ} 8.080$, de 19 de setembro de 1990. Dispõe sobre as condições para a promoção, proteção e recuperação da saúde, a organização e o funcionamento dos serviços correspondentes e dá outras providências. Diário Oficial [da] República Federativa do Brasil, Poder Executivo, Brasília, DF, v. 1, n. 81, 20 setembro 1990. Seção 1, p. 1-2.

[2] Brasil. Ministério da Saúde. Política Nacional de Gestão de Tecnologias em Saúde /Ministério da Saúde, Secretaria de Ciência, Tecnologia e Insumos Estratégicos, Departamento de Ciência e Tecnologia. - Brasília : Ministério da Saúde, 2010c. 48 p. (Série B. Textos Básicos em Saúde).

[3] Brasil. Ministério da Saúde. Fundação Oswaldo Cruz. Programa Farmácia Popular do Brasil: manual básico / Ministério da Saúde, Fundação Oswaldo Cruz. - Brasília: Editora do Ministério da Saúde, 2005. 102 p.: il. - (Série A. Normas e Manuais Técnicos).

[4] Brasil. Ministério da Saúde. Disponível em: http://www.pns.icict.fiocruz.br/. Acesso em: dezembro de 2013.

[5] Departamento de Informática do Sistema Único de Saúde (DATASUS). Sistema de Informações da Saúde. [banco de dados on-line]. Disponível em URL:http://datasus.saude.gov.br/sistemas-e-aplicativos/epidemiologicos/hiperdia. Acesso: julho de 2016.

[6] Brasil. Lei $\mathrm{n}^{\circ} 12.401$, de 28 de abril de 2011. Altera a Lei no 8.080, 19 de setembro de 1990, para dispor sobre a assistência terapêutica e a incorporação de tecnologia em saúde no âmbito do Sistema Único de Saúde - SUS. Diário Oficial [da] República
Federativa do Brasil, Poder Executivo, Brasília, DF, v. 1, n. 81, 29 abril 2011a. Seção 1, p. 1-2.

[7] Bronzino, JD. The Biomedical Engineering Handbook . 2. ed. USA : CRC Press, Inc, 1995).

[8] Brasil. Lei n ${ }^{0}$ 10.858, de 13 de abril de 2004. Autoriza a Fundação Oswaldo Cruz - Fiocruz a disponibilizar medicamentos, mediante ressarcimento, e dá outras providências. Diário Oficial da República Federativa do Brasil, Brasília (DF), 2004 abr 14; Seção 1:1.

[9] Pereira MA. Programa Farmácia Popular no Brasil: uma análise sobre sua relação com o complexo econômico-industrial da saúde e os programas estratégicos do governo federal [dissertação]. Rio de Janeiro (RJ): Escola Nacional de Saúde Pública, Fundação Oswaldo Cruz; 2013.

[10] Brasil. Ministério da Saúde. Glossário do Ministério da Saúde: Projeto terminologia em saúde. Brasília: Ministério da Saúde, 2004. [Acesso: Julho de 2016]. Disponível em: http://dtr 2001.saude.gov.br/editora/produtos/livros/pdf/04_06 44_M.pdf.

[11] Sociedade Brasileira de Diabetes (SBD). Tratamento e acompanhamento do diabetes mellitus. Diretrizes da Sociedade Brasileira de Diabetes, 2015-2016. Disponivel em: http://www. diabetes .org.br/sbdonline/images/docs/DIRETRIZES-SBD2015-2016.pdf. Acesso: Julho de 2016.

[12] International Diabetes Federation. IDF Diabetes Atlas [Internet]. 6a ed. Brussels: International Diabetes Federation, 2014. Disponível em: . Acesso em: Julho de 2016.

[13] World Health Organization. The World Health Organization Report 2002: reducing risks, promoting healthy life. Geneve: WHO, 2002.

[14] Brasil. Ministério da Saúde. Secretaria de Vigilância em Saúde. Sistema de Informações sobre Mortalidade. Disponível em: . Acesso em: Julho de 2016.

[15] Instituto Brasileiro de Geografia e Estatística. Pesquisa Nacional de Saúde: 2013. Percepção do estado de saúde, estilos de vida e doenças crônicas [Internet]. Rio de Janeiro: Instituto Brasileiro de Geografia e Estatística; 2014 [citado 2014 dez 22]. Disponível em: ftp://ftp.ibge.gov.br/PNS/2013 /pns2013.pdf. 